\title{
Educación, inclusión social y digitalización del aprendizaje: EpDLab como estudio de caso durante la crisis del COVID-19 \\ Education, social inclusion and digitization of learning: EpDLab as a case study during the COVID-19 crisis
}

\author{
Roi Guitián, María Lobo \\ epd@agareso.org, participacion@agareso.org \\ Educación para la Transformación Social \\ AGARESO \\ Santiago de Compostela, España
}

\begin{abstract}
Resumen- Durante el año 2020, las instituciones educativas tuvieron que adaptar toda su actividad docente, de forma imprevista, a modelos de aprendizaje virtual en un contexto de emergencia sanitaria. En el marco de la ESO, este proceso de adaptación era clave para seguir garantizando la educación como derecho fundamental de la ciudadanía. En este trabajo, exponemos los análisis, las conclusiones y las adaptaciones realizadas en el marco de un proyecto de Educación para la Ciudadanía Global. Entendemos la educación como eje vertebrador de la inclusión social y de la igualdad de oportunidades y, a través de un estudio de caso y de su comparación con diferentes informes nacionales y europeos, queremos poner el foco en la digitalización del aprendizaje como un factor determinante para la equidad, la igualdad de oportunidades, la alfabetización digital y mediática y la reducción de las desigualdades, ya que no es solo un proceso tecnológico, sino también pedagógico y social.
\end{abstract}

Palabras clave: Aprendizaje en línea, equidad, inclusión social, Politicas y estrategias educativas, Educación en tiempos de Covid19, digitalización de la enseñanza.

\begin{abstract}
In 2020, educational institutions were unexpectedly forced to adapt their teaching activities to digital learning strategies in a context of health emergency. In Secondary Education, this process of adaptation revealed as a key to guarantee education as a fundamental right for citizens. In this article, analysis, conclusions and adaptations will be exposed, framed in an Education for Global Citizenship project. Understanding that education is a main axis for social inclusion and for equal opportunities, and using a case of study in comparison with national and European reports, this research aims to highlight learning digitalization as a key factor for equity, equal opportunities, digital and media literacy and reducing inequalities, given that it is not only a technological but also a pedagogical and social process.
\end{abstract}

Keywords: Online learning, equity, social inclusion, educational policies and strategies, education in times of Covid-19, digitalization of teaching.

\section{INTRODUCCIÓN}

Este trabajo expone los resultados de nuestro estudio sobre el impacto de la digitalización del proceso de enseñanzaaprendizaje, a partir de nuestra experiencia en un caso práctico: EpDLab, un proyecto de Educación para la Ciudadanía Global (en adelante ECG) implementado de forma transversal en la programación de Educación Secundaria Obligatoria (en adelante ESO). En nuestro análisis, hemos relacionado aspectos tecnológicos (infraestructuras, acceso a dispositivos) con aspectos educativos (desarrollo de competencias y habilidades, estrategias pedagógicas) y factores relacionados con la desigualdad social (colectivos vulnerables o en riesgo de exclusión), describiendo los retos identificados y aportando una serie de recomendaciones basadas en las conclusiones obtenidas.

Como referencias, en este artículo usamos varios informes de resultados obtenidos de la consulta pública abierta (Open Public Consultation, en adelante OPC) sobre educación digital, así como otros análisis elaborados por la Comisión Europea en el marco del Digital Action Plan 2021-2027. A nivel nacional, son relevantes los resultados del CIS sobre "Tendencias en la Sociedad Digital durante la pandemia del COVID-19”, así como el diagnóstico y las líneas de actuación propuestas en el Plan Nacional de Competencias Digitales, en línea con el Plan España Digital 2025. Nuestra investigación se basó en la comparación e interrelación de estas publicaciones con los resultados obtenidos a partir de nuestro caso de estudio concreto, con el objetivo de ofrecer un diagnóstico y unas propuestas de actuación con respecto a la educación digital o $e$ learning, entendida como un factor clave en la construcción de una sociedad justa y equitativa, en garantizar la igualdad de oportunidades y en la lucha contra la exclusión social.

\section{CONTEXTO}

En marzo de 2020 se declaró en España el estado de alarma. Entre otras cosas, esta orden impuso el cierre de todos los centros educativos, lo cual obligó a los equipos docentes a adaptar todo su itinerario didáctico a un entorno virtual. La toma de decisiones y la elaboración de alternativas pedagógicas tuvo que hacerse a contrarreloj, sin pruebas previas o adaptaciones a situaciones particulares. En nuestro caso, como personal técnico que trabaja en el área de ECG, la exigencia era similar: nuestras actividades están integradas de forma transversal en el currículum y en la programación de los centros educativos con los que trabajamos. 
EpDLab, además de trabajar competencias relacionadas con los derechos humanos, el pensamiento crítico o el consumo responsable, tiene otro eje vertebrador: la competencia digital, una de las competencias claves reconocidas en la LOMCE, con el objetivo de educar en el acceso a los medios de información y comunicación (TIC) para el ejercicio de una ciudadanía plena. Su contribución a los estándares de la Educación Secundaria Obligatoria (ESO) aparecen descritos en "Contribución de EpDLab a los estándares de aprendizaje en la ESO, 2020", analizando esta contribución específica en las áreas de Lengua Gallega, Lengua Española y Valores Éticos. Este trabajo se desarrolla a lo largo de todo el calendario escolar integrado en diferentes materias, y en coordinación con el equipo docente y con el proyecto educativo de cada centro

En este contexto, la adaptación a un entorno virtual no parecía implicar un gran obstáculo. La mayoría de las competencias, objetivos didácticos y contenidos trabajados son coherentes con el aprendizaje a través de aulas virtuales. Además, el equipo de ECG de AGARESO, ONG que coordina el proyecto EpDLab, participaba en la elaboración de herramientas y metodologías de aprendizaje en línea en un proyecto europeo, contando con un amplio abanico de recursos para llevar a cabo esta adaptación. El profesorado necesitaba conocer y poner en práctica estos recursos, con el objetivo de adaptar su docencia al nuevo contexto. El alumnado necesitaba mantener el vínculo con la comunidad educativa, tanto a nivel de aprendizaje como de socialización. Vivíamos en confinamiento e Internet apareció como la herramienta para superar las restricciones espacio-temporales.

Este trabajo tiene un doble objetivo: por un lado, analizar el impacto de la digitalización en el aprendizaje, a partir de los cinco grupos de ESO participantes en un proyecto de ECG a lo largo de todo el curso escolar, y que cuenta con la participación de profesorado y alumnado de centros educativos situados en contextos diversos, tanto a nivel demográfico, como socioeconómico; de forma indirecta, el proyecto también requiere la participación de otros agentes relevantes, como el claustro y las instituciones públicas autonómicas. Por otro lado, y desde las conclusiones obtenidas, este trabajo ofrece, una serie de recomendaciones $\mathrm{y}$ estrategias dirigidas especialmente a instituciones públicas y privadas, a equipos de gestión política y educativa, a profesionales de la educación y, en general, a la comunidad educativa en el sentido más amplio, ya que este proceso de digitalización debe contemplar las consecuencias de su introducción en las aulas a nivel de aprendizaje, pero también garantizando la igualdad de oportunidades en el acceso a la educación obligatoria.

\section{DESCRIPCIÓN}

Este trabajo se ha realizado en dos fases: una primera fase durante el estado de alarma iniciado en marzo y hasta el final de curso en junio de 2020, en la que se realiza un diagnóstico y se identifican las necesidades. La segunda fase incluye el curso escolar completo desde septiembre de 2020 hasta junio de 2021, en la que se aplica un modelo de aprendizaje mixto (blended learning) en base a los resultados de la primera fase. Estas dos fases permiten, además, analizar los resultados en dos grupos de estudio: un grupo experimental, correspondiente al curso
2020-21, y un grupo de control, el del curso 2019-20. Ambos grupos pertenecen a los mismos centros y en la misma etapa educativa ( $\left.2^{\circ} \mathrm{ESO}\right)$, pero mientras que el grupo experimental participó en el proyecto con una metodología mixta o blended learning, en horario lectivo y con dispositivos y recursos facilitados por el centro para todo el alumnado, el grupo de control siguió una metodología completamente digital, sin tiempos marcados y con los dispositivos y recursos que cada familia pudiera aportar a este proceso. Además, esto permite analizar también el valor de los protocolos de anticipación a situaciones imprevistas.

\subsection{Diagnóstico y retos identificados}

En marzo de 2020, la crisis del COVID-19 provocó que reelaboramos todo el itinerario didáctico a través de una plataforma específica para aulas virtuales (Moodle), de herramientas de comunicación en línea y de contenidos interactivos para adaptar nuestras estrategias a un modelo de aprendizaje a distancia en entornos digitales (Padlet, Youtube, H5P, Mentimeter, Google Forms...) Durante la implementación de este modelo virtual, observamos los siguientes retos:

1. Nuestro modelo pedagógico se basa en el aprendizaje social: el aprendizaje entre iguales (P2P, peer-to-peer) como eje vertebrador. $\mathrm{El}$ hecho de tener a cada persona del grupo en un lugar diferente, en un dispositivo individual y con la posibilidad de desconectar en cualquier momento por motivos ajenos a la actividad impedían que se generasen interacciones imprescindibles para el aprendizaje en grupo.

2. El vínculo con el proceso de aprendizaje requiere que este sea significativo, es decir, que el alumnado identifique su relevancia en su contexto inmediato. Al no compartir un espacio físico con el resto del grupo, al igual que con la persona docente, este vínculo se debilita: el aula virtual no fue entendida por el alumnado como un espacio paralelo al centro educativo, sino que percibe Internet como un espacio de entretenimiento, de socialización lúdica y de ocio.

3. Otro factor clave son las competencias digitales del alumnado. Así lo explica una de las tutoras en su informe de evaluación: "los nativos digitales [...] no son tal cuando se trata de determinadas habilidades básicas: enviar correctamente un correo electrónico, moverse por un aula virtual, participar en una videoconferencia... Son habilidades que para algunos se convierten en una brecha casi insalvable para mantener el proceso educativo. Y no olvidemos, por favor, que no todas las familias tienen los mismos recursos materiales y tecnológicos, ni la misma capacidad para apoyar a sus hijos en este tipo de actividades"(memoria adjunta a informe de evaluación, 2020). Observamos también que el alumnado no estaba preparado para el aprendizaje autónomo. A pesar de que las actividades fueron diseñadas con contenidos interactivos, vídeos y documentos explicativos para ser realizadas de forma asíncrona, el alumnado no pudo seguirlas sin la guía y coordinación de una persona docente, en un horario determinado y con instrucciones cerradas: esperaban una estructura similar a la de las clases presenciales.

4. El acceso a dispositivos electrónicos por parte del alumnado apareció como otro factor determinante. Según el 
CIS, en la mayoría de los hogares estos dispositivos son compartidos por dos personas, y solo en el $21,5 \%$ de los casos hay un dispositivo para cada persona (CIS, 2021, p.5). En nuestro caso de estudio, esto se tradujo en un ordenador o tablet compartida, es decir, que parte del alumnado tenía que adaptarse a los horarios de otra $u$ otras personas.

5. En lo que se refiere a infraestructuras y recursos tecnológicos, la cobertura de red y el acceso a dispositivos electrónicos son dos de las fortalezas, identificadas como tales en las investigaciones del Digital Action Plan y en el Plan Nacional de Competencias Digitales para España. Sin embargo, tanto este informe como el europeo reconocen que existe una gran disparidad entre territorios: a nivel europeo, señalan las diferencias entre países y también entre territorios rurales y urbanos (Working document, Digital Action Plan, 2021, p.p. 26-29), y a nivel español, "la falta de equipamientos y la baja capacitación digital de buena parte de la población, con particular incidencia [...] en el ámbito de la educación y en las PYMEs" (Plan Nacional de Competencias Digitales, 2021, p.3) Nuestras conclusiones se acercan más al diagnóstico a nivel español: entre los 5 centros educativos del proyecto, el más alejado de núcleos urbanos contó con el equipamiento, la cobertura de red y las habilidades para adaptarse al entorno virtual: "en este sentido, las instituciones educativas que tenían experiencia en organizar la enseñanza, el aprendizaje y la evaluación como un trabajo en equipo con un enfoque organizacional fueron capaces de adaptarse fácilmente a la educación a distancia"(Jenavs y Strods, citados en Digital Action Plan, 2020, p.34). Este centro se ubica en una pequeña población rural, donde equipo docente y alumnado comparten no solo el espacio de aula, sino otros espacios comunitarios; así, fueron capaces de articular una red de apoyo para aquellos estudiantes con más dificultades de aprendizaje o con menos recursos para acceder a los dispositivos electrónicos.

6. Los centros educativos y el personal docente respondieron de distintas formas a esta situación. Una gran parte no contaban con los conocimientos ni con la experiencia para adaptar su docencia a un entorno digital, y vieron como su carga de trabajo se multiplicó, con lo que perdieron el contacto con el alumnado y/o reprodujeron modelos presenciales con recursos digitales (Working document, Digital Action Plan, 2021, p.34). Según el barómetro del CIS publicado en marzo de 2021, casi un $92 \%$ de los estudiantes cree que "el centro educativo tuvo que improvisar métodos y protocolos online" (CIS, 2021, p.32). En nuestro caso de estudio, cuatro centros optaron por reproducir dinámicas de la educación presencial mediante videoconferencias y actividades síncronas y solo uno de los centros manifestó sentirse preparado para afrontar la situación (citado en punto 5). En este proceso, perdimos el contacto con dos grupos: uno de ellos volvió a unirse al proyecto más adelante, ya que no tenía contacto con su tutor desde el cierre de las escuelas. Este tutor manifestó no estar preparado para adaptar su docencia a una estrategia online, pero facilitó nuestra tarea para retomar nuestro trabajo educativo con su alumnado. El segundo grupo con el que perdimos el contacto responde a otras razones que serán expuestas en el siguiente punto: el factor socio-económico.

7. Según los datos del CIS, el $87 \%$ de los hogares españoles cuentan con conexión a Internet, y solo el $12 \%$ reconoce no tener buena cobertura de red; además, hay dispositivos electrónicos (ordenador, portátil o tablet) en casi el 94\% de ellos (CIS, 2021, p.4-5). Al cruzar estos datos con factores socioeconómicos como la ocupación, los resultados muestran que la mayoría de los hogares que no cuentan con Internet y/o con dispositivos electrónicos se corresponden con el sector primario, el trabajo doméstico no remunerado y las personas en situación de inactividad laboral diferente al desempleo (además de las personas jubiladas, que también representan un alto porcentaje) (CIS, 2021). Al preguntar por las razones, los porcentajes más altos se encuentran en la falta de competencia digital: la mayoría reconoce no contar con las habilidades necesarias para hacer uso de la red. En este tema que nos ocupa, relacionamos estos resultados con las conclusiones del Digital Education Action Plan de la UE: "la situación socioeconómica de las familias jugó un papel crucial [...] Los padres y madres con mayor formación estaban mejor posicionados para ayudar a sus hijos e hijas para sostener un ambiente educativo en casa" (Digital Action Plan, 2020, p.7)

Estos resultados coinciden con nuestro diagnóstico: un grupo-clase perdió completamente su vínculo con el proceso de aprendizaje siendo fundamental el factor socio-económico ya que sus familias, pertenecientes al tramo de ingresos más bajo o en situación de desempleo y con un bajo nivel de formación, no pudieron apoyar a sus hijos/as en este proceso. El resultado fue la completa desvinculación de un alumnado con importantes necesidades de apoyo: se trataba de un grupo PMAR (Programa de Mejora del Aprendizaje y del Rendimiento). Citando de nuevo el informe de evaluación de la tutora de este grupo: "Llegó la suspensión de las clases y con las clases finalizó EpDLab en este grupo. Y no finalizó porque el proyecto no se había adaptado o porque no se les había informado de su recorrido en la red. Finalizó porque, como dije al principio, con este alumnado la docencia presencial, el contacto humano vis a vis no es importante, es imprescindible. Están muy lejos de ser totalmente autónomos en su proceso de aprendizaje, en muchos casos hay una falta notable de medios técnicos propios, y su motivación desciende drásticamente cuando salen del aula" (memoria adjunta a informe de evaluación, 2020). Esto significa que un 9\% de nuestro alumnado no pudo continuar con su proceso educativo durante el cierre de 2020, coincidiendo con aquel alumnado que partía de una situación socio-económica más desfavorable, tanto en lo que se refiere al la capacidad familiar de darles apoyo educativo como en las posibilidades de acceso a Internet y a dispositivos electrónicos en casa.

\subsection{Actividades, metodologías y recursos.}

A partir de este diagnóstico, creemos que la adaptación de la enseñanza a entornos digitales es al mismo tiempo un reto y

una oportunidad. Aún así, queremos poner el acento en lo primero, ya que nuestra observación de los resultados y de los procesos en sí mismos nos llevan a una serie de estrategias concretas para aprovechar el impacto positivo de este cambio.

El aprendizaje es siempre un proceso social, en cualquier etapa, y en la OPC citada en este artículo, el colectivo de estudiantes reconoció que la comunicación con el resto de la comunidad educativa fue la principal necesidad no cubierta durante la crisis del COVID-19; el Digital Action Plan 
recomienda así la educación semi-presencial o blended learning, y reclama el apoyo de las instituciones para asegurar una dinámica fluida y orgánica entre el aprendizaje en línea y el presencial (Working Document, Digital Action Plan, p.76 y 81-82). En España, el barómetro del CIS muestra resultados similares, ya que la mayoría de estudiantes respondieron que, aunque la educación presencial es imprescindible, deberían incorporarse algunas actividades online, (CIS, 2021, p.20).

En EpDLab, siguiendo estas recomendaciones y partiendo de los resultados de la primera fase, optamos por una modalidad mixta de aprendizaje para el curso 2020-21: combinamos un aula virtual, con contenidos interactivos y multimedia, con dinámicas presenciales, como por ejemplo, actividades de debate $\mathrm{o}$ de producción de contenidos audiovisuales. Aseguramos, así, el desarrollo de competencias digitales a través del e-learning al tiempo que garantizamos la socialización presencial, que facilita y mejora de forma notable la calidad de las comunicaciones con el alumnado, el profesorado y el centro educativo. Ante la incertidumbre previa al curso escolar 20-21, nuestro equipo técnico optó por diseñar una programación didáctica que contemplase diferentes escenarios y se adaptó a las nuevas restricciones sanitarias con las siguientes estrategias:

1. Los materiales didácticos y las dinámicas fueron modificados en su totalidad: optamos por evitar el trabajo con materiales físicos compartidos (cartulinas o post-it) y buscamos herramientas que nos permitieran trasladar estas dinámicas, así como los contenidos, a un entorno virtual. En este sentido, mantenemos Moodle como plataforma de alojamiento para el aula virtual (hasta el momento, es la que mejor nos garantiza la estabilidad y la seguridad con respecto a los datos del alumnado) y sesiones estructuradas con vídeos, contenidos interactivos y herramientas de trabajo colaborativo.

2. En línea con las recomendaciones del Digital Action Plan 2021-2027, optamos por una estrategia de blended learning: la mitad de las sesiones son presenciales, aunque se desarrollan con materiales y contenidos ubicados en el aula virtual, y la otra mitad son virtuales, desarrollándose sin nuestra presencia en el aula, con nuestro soporte a distancia. Así, apoyamos el desarrollo de habilidades de aprendizaje autónomo en entornos digitales, atendiendo a lo recogido en la evaluación de la experiencia durante el curso pasado donde: "una amplia mayoría del profesorado coincide en señalar que, si bien no contemplan que EpDLab se pueda desarrollar en el entorno virtual con los mismos resultados satisfactorios que en las aulas, sí podría ser viable la implementación de una modalidad mixta, siempre y cuando: "se prepare antes al alumnado se habiliten canales de participación y seguimiento en tiempo real con la presencia del personal de AGARESO" (informe de evaluación externa de EpDLab,2020,p.36).

3. El desarrollo de las competencias digitales del profesorado $\mathrm{y}$ de los equipos gestores de las instituciones educativas debe ir más allá de la formación en herramientas o plataformas concretas: los profesionales son la guía en este proceso de digitalización, y "necesitan contar con las competencias y la confianza necesarias para usar la tecnología de forma efectiva" (Caena y Redecker, citado en Digital Action Plan, 2020, p.34). En nuestro trabajo, hemos introducido dos herramientas que pueden apoyar este proceso: por un lado, una formación dirigida a docentes y profesionales de la educación con el objetivo de aportar marcos teóricos, estrategias pedagógicas y herramientas de trabajo en entornos digitales, en paralelo al desarrollo de la competencia mediática en el aula (hemos observado una importante demanda por parte del profesorado para trabajar el pensamiento crítico y el tratamiento de la información, principalmente abordado desde los bulos o noticias falsas y el discurso de odio). Por otro lado, también hemos coordinado una prueba de nuestra aula virtual en centros ajenos al proyecto que nos ocupa, con la finalidad de recibir aportaciones y mejoras por parte del profesorado sobre nuestra propuesta, así como evaluar el impacto de estas adaptaciones en grupos sin formación previa al respecto.

4. Las actividades se desarrollan en horario lectivo: de esta forma, se asegura el acceso a equipamientos electrónicos con conexión a Internet para cada persona. En los centros participantes, gracias a programas específicos de cesión de equipos del gobierno autonómico, cada estudiante cuenta con un dispositivo de uso individual. En el caso de aquellos centros adheridos al programa E-Dixgal, el alumnado cuenta con portátiles que, en caso de necesidad, pueden llevar a sus casas. Por otro lado, el apoyo al proceso educativo no recae en la familia o en las personas cuidadoras, sino que todo el grupo cuenta con el mismo apoyo de forma igualitaria, eliminando factores de desigualdad basados en la situación socioeconómica o en el nivel de alfabetización, y garantizando el acceso a una educación universal y de calidad.

\section{RESUlTADOS}

El éxito de la integración del e-learning no depende únicamente de las infraestructuras, del acceso a equipamiento, de la competencia digital de profesorado y alumnado o de la adecuada traducción de las pedagogías a un entorno mixto, sino a todos estos factores interrelacionados como partes de una misma realidad: "no consiste únicamente en replicar o trasladar prácticas presenciales o propuestas tradicionales. Es un proceso complejo, que requiere una robusta competencia digital, que incluya el cambio organizacional, el seguimiento continuo y la orientación hacia pedagogías centradas en el aprendizaje" (Working Document, Digital Action Plan, 2020, p.26). En nuestro caso de estudio, un centro rural de interior, alejado de núcleos urbanos, fue el que mejor respondió a esta adaptación, bajo un modelo integral donde el acceso a los recursos tecnológicos, la organización del trabajo en equipo, la competencia digital de la comunidad y la comunicación efectiva entre sus miembros fueron abordados como elementos del mismo proceso de enseñanza-aprendizaje. El impacto de esto es también integral, no solo a nivel educativo, sino social.

Para evaluar este impacto, utilizamos herramientas de evaluación interna y una evaluación externa. Respecto a la primera, se trata de una evaluación continua de carácter cuantitativo (registros en el aula virtual, cuestionarios, tiempo de conexión, finalización de actividades) como cualitativas (dinámicas de reflexión colectiva y design-thinking, reuniones periódicas con el equipo docente, memorias). Por otro lado, cada curso escolar este proyecto es evaluado por un agente 
externo que mide el cumplimiento de los indicadores formulados y el impacto de la acción a nivel grupo-clase.

\section{CONCLUSIONES}

El modelo descrito para la adaptación del aprendizaje a entornos digitales responde a las recomendaciones de varios planes de digitalización del aprendizaje en los que se reconoce la educación como un derecho que debe garantizar la igualdad de oportunidades y en las tecnologías digitales como un posible factor de desigualdad y de exclusión social: "la brecha digital entre regiones y entre grupos socio-económicos distintos sigue siendo un gran obstáculo y debería tener un lugar prioritario en la agenda política: la competencia y la alfabetización digital [...] deben desarrollarse en paralelo a la infraestructura. Solo de esta forma, las inversiones en tecnología demostrarán ser eficientes"(Karpiński., Di Pietro., Castaño Muñoz y Biagi, 2020, p.77). De este modo para garantizar la sostenibilidad y la replicabilidad de este trabajo, EpDLab se apoya en las siguientes estrategias y acciones:

- Publicaciones que incluyen las unidades didácticas y las guías de uso de las mismas tanto en modalidad presencial como virtual, disponibles para libre descarga desde la web del proyecto bajo licencia CC-BY-SA.

- Acceso abierto a las aulas virtuales y a sus contenidos con permisos de usuario invitado, en las que solo se restringe el acceso a los datos personales del alumnado y el profesorado participante.

- Cursos de formación dirigidos al profesorado y a otros profesionales de la educación, que integran y ponen en práctica las estrategias didácticas y de adaptación al contexto posterior a la crisis del COVID -19.

- Actividades y talleres concretos para otros centros educativos y para agentes del tercer sector que trabajen en ECG, centrados en el desarrollo de habilidades digitales, la competencia mediática y el aprendizaje autónomo.

Así, nuestra principal conclusión y la recomendación derivada de ella implica que la educación debe ser entendida como un eje vertebrador de la inclusión social y la igualdad de oportunidades; centrándonos en la digitalización de los procesos de aprendizaje, creemos que todas las conclusiones y recomendaciones expuestas en este trabajo están alineadas con los resultados del Digital Action Plan europeo, con los de la Agenda 2030 y con los Objetivos de Desarrollo Sostenible (ODS), así como con el Plan Nacional de Competencias Digitales que proponen "una hoja de ruta para la construcción de un mundo más justo y sostenible y reconoce la necesidad de adquirir, desarrollar y utilizar competencias digitales para, respectivamente, contribuir al fin de la pobreza (ODS 1), garantizar una educación inclusiva y equitativa de calidad (ODS 4), [...] la reducción de las desigualdades (ODS 10) (Plan Nacional de Competencias Digitales, 2021, p.3-4).

La elaboración de propuestas políticas concretas siguiendo esta "hoja de ruta" se evidencia como imprescindible al presentar las conclusiones de nuestro caso de estudio, EpDLab: cuando la continuidad del alumnado dependía únicamente de la situación socio-económica familiar, la desigualdad y los factores de exclusión se vieron acentuados y, en algunos casos, se perdió por completo el vínculo con el sistema educativo. La continuidad de este vínculo dependió casi únicamente de si tenían o no conexión a Internet en casa y/o dispositivos como ordenadores, tablets o portátiles, si podían hacer uso de estos dispositivos con libertad o si tenían que compartirlos, si sus padres, madres o personas cuidadoras contaban con la formación y las herramientas pedagógicas necesarias para acompañar el proceso, y de si el centro educativo podía mantener la comunicación continua para suplir las carencias observada. Sin embargo, una vez comenzaron las clases presenciales, las aulas ofrecieron un contexto igualitario, y nuestras sesiones responden a una modalidad semi-presencial acompañado en todo momento por docentes, facilitando los intercambios grupales y la creación colectiva mientras que, por otro lado, desarrolla todo el itinerario didáctico a través de contenidos interactivos, herramientas digitales y estrategias pedagógicas adaptadas a plataformas o aulas virtuales.

\section{Tabla 1}

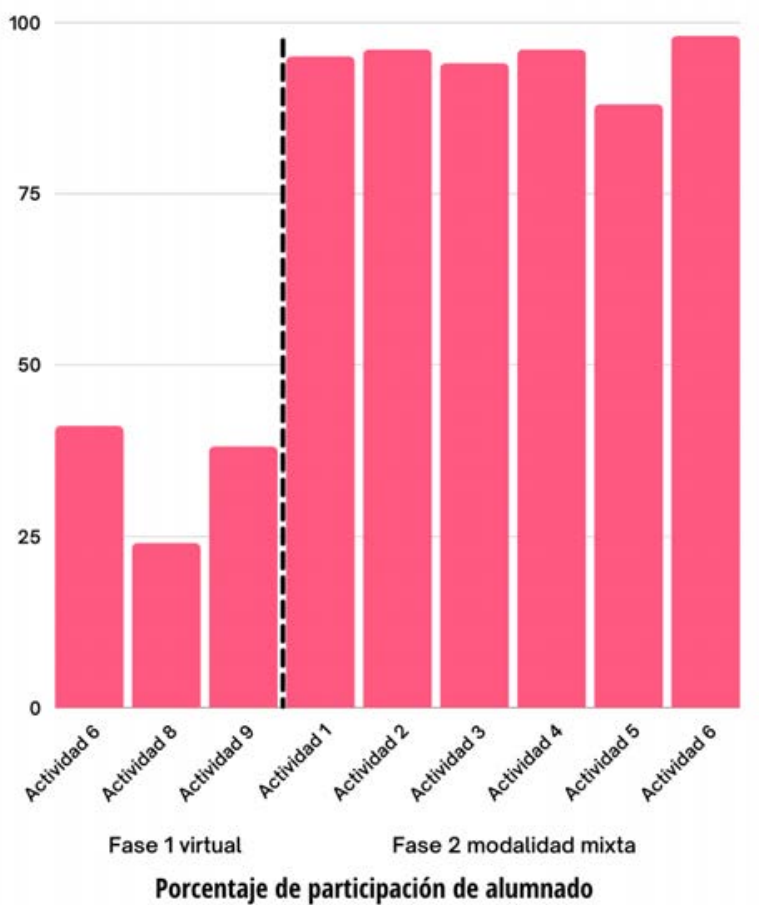

Aún así, creemos que esta situación implica demasiados riesgos, teniendo en cuenta todo lo aprendido durante la crisis del COVID-19: el acceso a la educación no puede depender de la situación socio-económica del alumnado o del centro educativo. Las competencias digitales del profesorado, de los equipos gestores, de las familias y de las personas cuidadoras son la clave para conseguir que nadie se quede atrás por razones económicas o sociales, pero también están en estrecha relación con el contexto socio-demográfico y la agenda política a nivel estatal, regional y local. Además de la formación en habilidades digitales, la Educación para la Ciudadanía Global es fundamental para reconocer el rol que la digitalización de la 
educación tiene desde una perspectiva integral, incluyendo su interdependencia con los ODS, la Agenda 2030, el Digital Action Plan europeo y el Plan España Digital 2025. A partir de nuestro trabajo de campo, seguiremos insistiendo en reivindicar la conectividad y el equipamiento adecuado, el acceso a plataformas enfocadas al aprendizaje para toda la vida y a herramientas digitales que apoyen este proceso, al tiempo que seguiremos poniendo en valor la digitalización de la enseñanza como un factor determinante para desarrollar la equidad, la igualdad de oportunidades, la alfabetización digital y mediática y la reducción de las desigualdades.

\section{REFERENCIAS}

AGARESO. (2020). Guía de uso de las actividades de EpDLab: contribución a los estándares de aprendizaje en la ESO y Guía de uso de las actividades online. Recuperado de https://epdlab.gal/guia-didactica/

Centro de Investigaciones Sociológicas - CIS. (2021). Tendencias en la sociedad digital durante la pandemia de la COVID-19 (Estudio 3316 y Estudio 3316/0-0, tabulación por ocupación) Recuperado de http://www.cis.es/cis/export/sites/default/-Archivos/Marginales/3300_3319/3316/es3316mar.pdf

European Commission. (2020). Public consultation on the new Digital Education Action Plan. Recuperado de https://ec.europa.eu/education/news/public-consultationnew-digital-education-action-plan_es

European Commission. (2020). Commission Staff Working Document, Digital Education Action Plan 2021-2027: Resetting education and training for the digital age. Recuperado de https://ec.europa.eu/education/sites/default/files/document-library-docs/deap-swd-sept2020_en.pdf

Gobierno de España. (2021). Plan Nacional de Competencias Digitales. Recuperado de https://portal.mineco.gob.es/RecursosArticulo/mineco/ministerio/ficheros/210127_plan_nacional_de_competencias_digitales.pdf

Karpiński Z., Di Pietro G., Castaño Muñoz J.and Biagi F. (2020). Digital Education Action Plan 2021-2027: Resetting education and training for the digital age, Summary of the Open Public Consultation

Ministerio de Educación y Formación Profesional, Gobierno de España, Currículo en Primaria, ESO y Bachillerato, Competencias Clave, recuperado de https://www.educacionyfp.gob.es/educacion/mc/lomce/curriculo/competencias-clave/competencias-clave.html

Wayna Consultora. (2020). Informe de evaluación externa de EpDLab y documentos anexos.

Bibliografía

Boud, D., Cohen, R. y Sampson, J. (2002). What is Peer Learning and why is it important?, Making the move to peer learning, in Peer Learning" en Higher Education: Learning From \& With Each Other. Recuperado de https://tomprof.stanford.edu/posting/418
Caena, F., \& Redecker, C. (2019). Aligning teacher competence frameworks to 21st century challenges: The case for the European Digital Competence Framework for Educators (Digcompedu). European Journal of Education, 54(3). doi:10.1111/ejed.12345

Jenavs E. y Strods J. (2020). Managing a school system through shutdown: lessons for school leaders. Edurio, Latvian Ministry of Education and Science, recuperado de: https://home.edurio.com/report-shutdown-lessons,

Marcos, L. y Ubrich T. (2016). Necesita mejorar: por un sistema educativo que no deja a nadie atrás, Save the Children. Recuperado de https://www.savethechildren.es/sites/default/files/imce/docs/necesita-mejorar-fracaso-escolar-savethechildren-ok.pdf

Guitián, R, Lobo, M. y Díaz, S. (2019). Narrativas digitales para potenciar la creatividad, la participación y el pensamiento crítico en la enseñanza obligatoria. Actas del V Congreso Internacional sobre Aprendizaje, Innovación y Cooperación. CINAIC 2019. doi: 10.26754/CINAIC.2019.0084 